\title{
Progress in high-energy models and impact for applications
}

\author{
A. Boudard ${ }^{\mathrm{a}}$ \\ CEA-DAPNIA-SPhN, CEN-Saclay, 91191 Gif-sur-Yvette, France
}

\begin{abstract}
A brief review of the models available to compute spallation reactions and associated observables is done in the context of potential applications. The success and main difficulties are discussed mainly from INCL4-ABLA calculations and developments, but trying to remain general for the main conclusions.
\end{abstract}

\section{Introduction}

Spallation processes are discussed in the context of accelerator driven systems, spallation neutron sources, or irradiation of vessels during space missions with impacts on electronics and human body. Having this type of applications in mind it is clear that we expect from the models a good predictive power for the light particles, especially the neutrons. The neutron absolute flux as a function of the neutron energy, and its emission angle (relative to the primary incident particle) but also to a smaller extent the energetic protons and pions produced in the first interaction which propagate in massive materials are needed and of first importance. The recoiling nuclei are also important in several specific aspects. Especially in the case of liquid targets (mercury, $\mathrm{Pb}-\mathrm{Bi}$, molten $\mathrm{U}$ or Th salts), a good knowledge of chemical species which will accumulate is needed to appreciate the chemical interactions with the containers. The $\mathrm{Z}$ dependence of the cross sections is there important. The recoil energy of the nuclear residues will produce damage in the solid materials (beam window, containers, transistors, etc.). This quantity is associated with the mass (or A) dependence of the cross section. The spallation target and the surrounding materials will be submitted to high flux of energetic particles and so will be activated by spallation processes. A good knowledge of all the cross sections for the isotopic production of spallation residues function of $A$ and $\mathrm{Z}$ is needed for a prediction of the materials activities after an irradiation. In activation problematic (as in chemical problems) although cross sections are the crucial relevant quantities, the largest impact is frequently not associated with the largest of them. Radio-nuclides with short periods (days) are important for target manipulations whereas long periods (thousand of years) are relevant for the evaluation of the waste harmfulness. For the radioprotection, volatile radioactive residues are obviously specifically important, and finally the rather high production of helium should be well under control since this gas, not diluted in metals can produce a swelling of the structures.

Basic tools to study a complex design submitted to a primary flux are transport codes like LAHET, MCNPX, FLUKA

\footnotetext{
${ }^{a}$ Presenting author, e-mail: alain.boudard@cea.fr
}

or GEANT4. They propagate the particles in the complex geometry and let them interact in the various media according to probabilities governed by the cross sections of the various possible outgoing channels. So these cross sections are basic ingredients which are either measured (for neutrons below $20 \mathrm{MeV}$ ) or obtained from physical models. In the last decade, campaign of systematic measurements have been driven especially under European projects to extend the experimental knowledge of cross sections up to $150 \mathrm{MeV}$. A concerted effort on the low energy theory of nuclear reactions based on optical potentials and coupled channel equations (codes like TALYS or GNASH) was also driven to complement the missing data.

As energy increases the number of possible outgoing channels is enormously multiplied and a systematic measurement for all possible targets becomes impossible. It is already the case that in the extended libraries up to $150 \mathrm{MeV}$, the knowledge of residues is rather poor. On the other side, all the tricky aspects of nuclear structure becomes less important and global predictive models not linked to a precise energy or target nature becomes possible. This is the place of the so called 'high energy' models of the spallation processes.

\section{Type of models}

High energy models are based on the assumption that the wave length associated with a nucleon of $\sim 1 \mathrm{GeV}$ is shorter than the inter-nucleon distance in the nucleus so that the nucleonnucleus interaction can be basically described by series of independent N-N interactions. This is the so called Intra Nuclear Cascade ([1] for a discussion of INC hypotheses). The elastic scattering is out of this scope but it is small and can be computed if needed by well adapted models like Glauber [2] or KMT [3]. The successive N-N interactions eject a few energetic particles (nucleons and pions) which carry most of the kinetic energy of the incoming nucleon and are relevant for the transport followed in complex designs. The remaining kinetic energy is then converted in excitation of the remnant nucleus $(\sim 150 \mathrm{MeV}$ out of $1 \mathrm{GeV}$ but with very high fluctuations from 0 to $\sim 800 \mathrm{MeV}$ ) the identity of which (A, Z and intrinsic spin $\mathrm{J}$ ) is determined by the INC. This generally highly excited nucleus decays by various processes: the evaporation of light 
particles (at least $\mathrm{n}, \mathrm{p}$ and ${ }^{4} \mathrm{He}$ are considered and possibly heavier ones) in competition with a possible fission of the remnant nucleus. More exotic decays are sometime considered like the multi-fragmentation of the nucleus when the excitation energy per nucleon exceed $\sim 3 \mathrm{MeV}$ or very asymmetric binary decays of the nucleus (also named Transition State Model).

INC models were originally treating the nucleus as a medium out of which the point like incident particle was, according to the $\mathrm{NN}$ scattering length promoting nucleons which were then explicitly followed. The codes ISABEL [4] and Bertini [5] found in LAHET and MCNPX are of that type. J. Cugnon with INCL [6] has introduced a more symmetric treatment with the explicit classical motion of all the nucleons of the target nucleus in the nuclear potential and a criteria for $\mathrm{NN}$ interactions based on the minimal distance of approach. The recent BRIC code of $\mathrm{H}$. Duarte [7] follows this scheme with in addition a realistic curvature of the trajectories in the potential. Originating from Nucleus-Nucleus reaction theory, QMD based cascades introduce an extension of the nucleon with Gaussian wave packets and a Skyrme interaction ([8] and communications $[9,10])$. As the nucleon energies become smaller during the cascade, INC justifications are weaker. So some authors introduce a "pre-equilibrium" phase before the de-excitation. The CEM codes of S. Mashnik (associated to the V. Barashenkov et al. cascade) are of that type [11]. In LAHET, the use of the Bertini cascade is also recommended associated with pre-equilibrium. Whether or not this stage introducing more flexibility but through more phenomenology is needed or partially included in the INC is still a question of interesting debates.

The typical de-excitation code is the old Dresner-Atchison one with an evaporation based on the Weisskopf-Ewing formulation. In the modern GEM [12] code, the parameters have been updated and the evaporation of particles extended up to ${ }^{24} \mathrm{Mg}$. The ABLA code [13] developed at GSI and actually part of an Abrasion-Ablation code, pay a special attention to the fission channel. In the present public version, only $\mathrm{p}, \mathrm{n}$ and $\alpha$ particles were treated in the evaporation but a new version (ABLA07 [14]) with more evaporated particles, new inverse cross sections and a refined fission treatment will be presented in this conference. The GEMINI code [15] treats the very asymmetric binary decays with the Transition State Model and the evaporation in the spin dependent formulation of Hauser-Feshbach. The SMM code [16] has a multi-fragmentation of the nucleus in addition to fission and evaporation.

\section{Possible precisions and difficulties}

Most of the codes already mentioned (INCL4 [17], ISABEL [4], CEM2k [11], BRIC [7]) give a rather precise account of cross sections in a wide domain of beam energies and for many targets. The double differential cross sections of neutron production for incident protons in the $\mathrm{GeV}$ range is generally correctly predicted (fig. 1). In the forward direction, the quasielastic peak appears slightly too high in energy and too narrow with INCL4. The excitation of the $\Delta$ resonance is correct whereas some versions of Bertini give an abnormally high contribution at $0^{\circ}$ [18]. The excitation energy at the end of the cascade must be correct for a good account of the isotropic

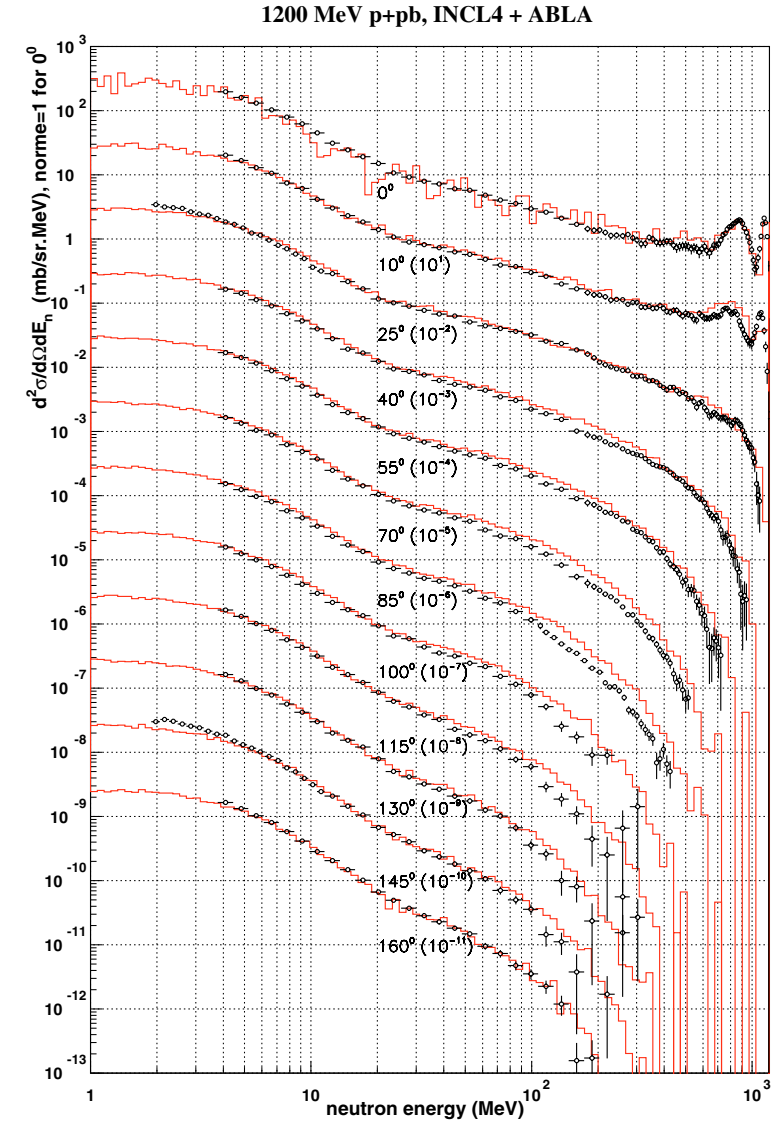

Fig. 1. Double differential cross sections for neutron production from protons $(1.2 \mathrm{GeV})$ on lead. The data [18] are the points. The histogram is an INCL4 calculation without clusters coupled with ABLA.

evaporative part below $20 \mathrm{MeV}$. The region $20 \mathrm{MeV}-100 \mathrm{MeV}$ is very delicate since the INC validity can be questioned there for such a low energy. In INCL4, these nucleons are contributing to the formation of cascade clusters. For thick targets (fig. 2), the neutron emission less structured is also quite correct as a function of energy and emission angle.

The cross section for the production of spallation residualnuclei is precisely computed for masses close to the target one. A systematic under prediction of light evaporation-residues is observed around $1 \mathrm{GeV}$ for heavy targets (lead for example) with INCL4 [17] as well as with ISABEL (fig. 3) whereas it is not the case at $500 \mathrm{MeV}$ [20] when a pure evaporation is coupled with the cascade. Similar difficulties are observed in the spallation of iron [21] and this is interpreted as the signature of additional de-excitation mechanisms linked with a large excitation at the end of the cascade. The experimental study of correlations between the fragments of iron spallation measured with the SPALADIN setup at GSI [22] already pointed out the relevance of the Transition State Model as treated by the GEMINI code. The precise recoil velocity spectra of ${ }^{136} \mathrm{Xe}$ fragments measured with FRS [23] exhibits two components which brings also valuable information on the various decay mechanisms of the de-excitation process.

At the moment, the ABLA code appears as the most coherent one to predict isotopic distributions of fission products and evaporation residues of a mass close to the target 


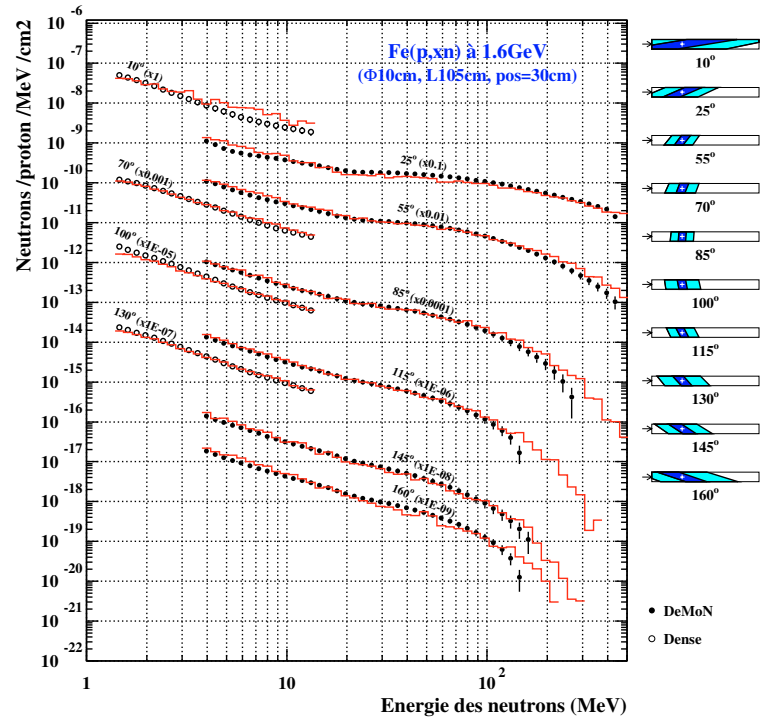

Fig. 2. Neutron production from a cylindrical $(105 \mathrm{~cm}$ long, diameter of $10 \mathrm{~cm}$ ) iron target with a $1.6 \mathrm{GeV}$ proton beam. The points are data [19], the histogram is an INCL4 calculation coupled with ABLA with transport treatment by LAHET. The schemes show which part of the target is seen by the detectors at each angle.

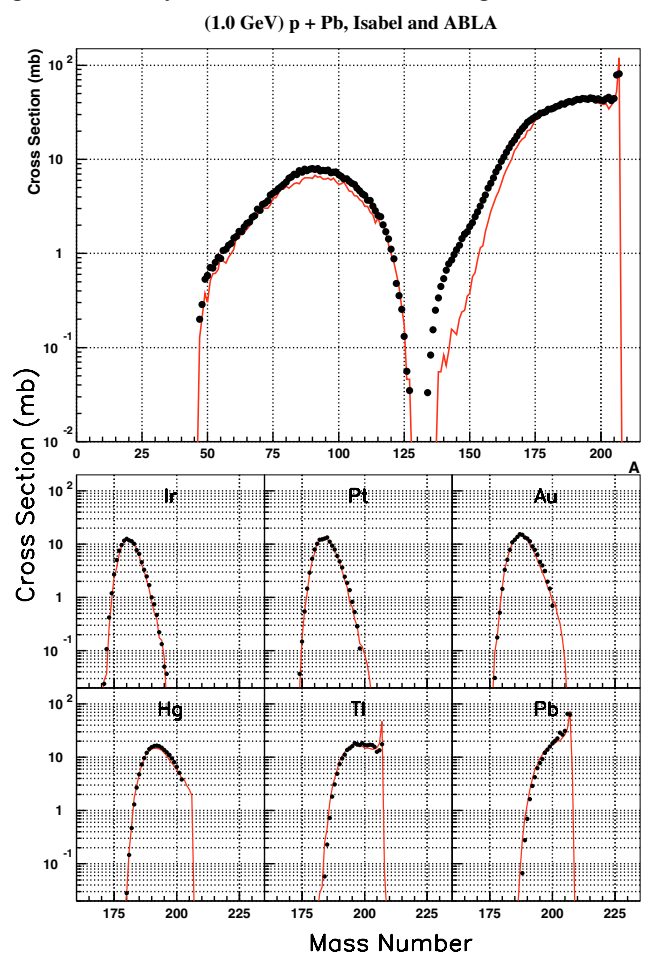

Fig. 3. Residual-nuclei production cross sections (data from [30]) compared with a Isabel-ABLA calculation (histogrammes). Isotopic cross sections are summed for constant mass number (top). Some isotopic families of heavy residues are displayed in the bottom part.

one, but edges of distributions remain rather imprecise and should be used with some care.

\section{Improvements of INCL4}

Compare to the public version INCL4.2 [17], the code (now version INCL4.4) has been improved in several ways.
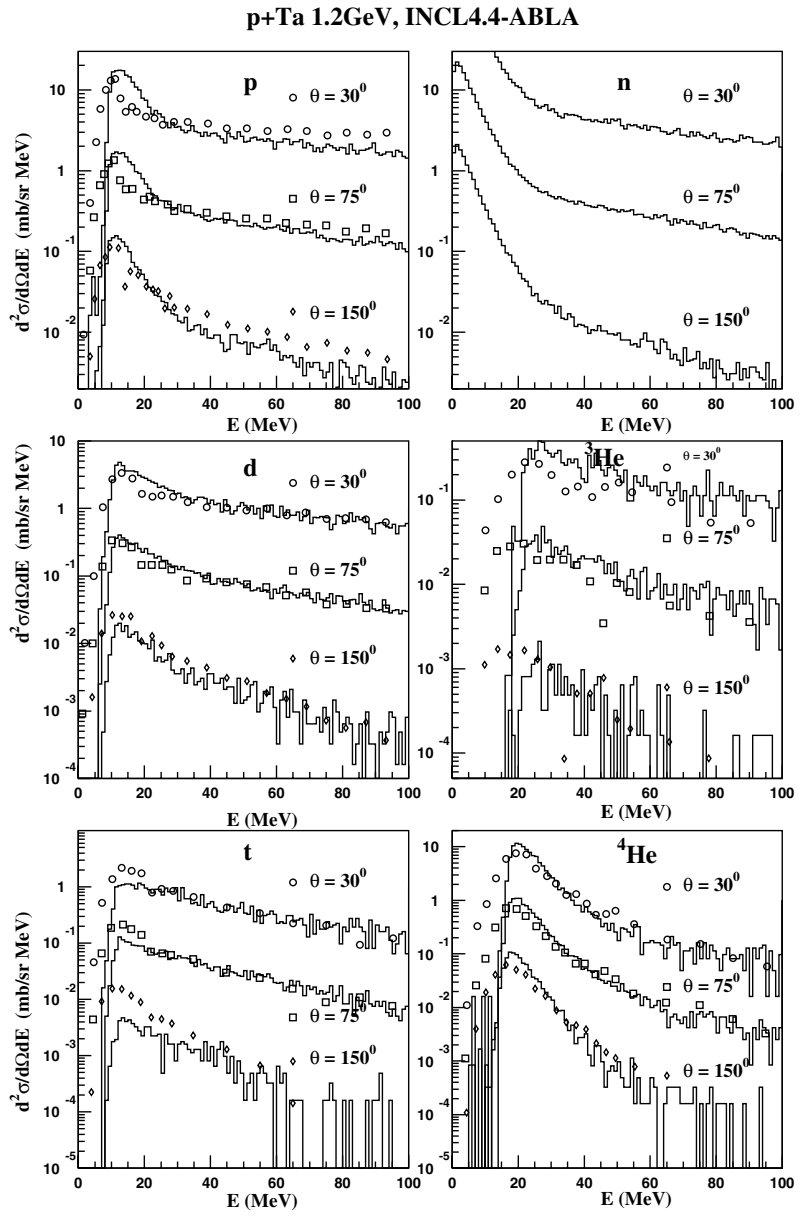

Fig. 4. Double differential cross sections of $p, n, d,{ }^{3} \mathrm{He}, \mathrm{t}$ and ${ }^{4} \mathrm{He}$ production from a Ta target with a $1.2 \mathrm{GeV}$ proton beam. Points are data from [27] and histograms are an INCL4.4 calculation including the last treatment of cluster production (see text) coupled with ABLA.

A dependence of the nuclear potential depth with the isospin of the baryon (nucleon or 433 ) based on the Fermi gas model has been introduced. For nucleons, a dependence with the kinetic energy has been also implemented, following optical potential systematics. The pions in the nucleus are now moving in an attractive phenomenological potential. This work is part of the Th. Aoust $\mathrm{PhD}$ [24] and is detailed in ref. [25]. Basically this improves the pion production and the nucleon cross sections at low energy.

The production of composite light particles $\left(d, t,{ }^{3} \mathrm{He}\right.$ and ${ }^{4} \mathrm{He}$ ) has been revisited following the original idea of a dressing of escaping nucleons at the surface of the nucleus [26]. The clusterization is done with a r-space, p-space vicinity criteria $(\Delta r * \Delta p<387 \mathrm{fm} . \mathrm{MeV} / \mathrm{c})$ and at a fixed distance from the mean nuclear radius $\left(R_{0}+2 \mathrm{fm}\right)$. The priority is given to the heaviest possible cluster and at random if a triton and $\mathrm{a}^{3} \mathrm{He}$ are possibly formed. Energy, including cluster binding energy, and momentum are conserved. The appropriate Coulomb barrier is also treated. Note that with only 2 empirical parameters this simple approach gives a reasonable account of the double differential cross sections up to $150-200 \mathrm{MeV}$ for incident neutrons of $513 \mathrm{MeV}$ on $\mathrm{Bi}$ and $\mathrm{Cu}$, protons of $2.5 \mathrm{GeV}$ on $\mathrm{Au}$ and protons of $1.2 \mathrm{GeV}$ on $\mathrm{Ta}$ (fig. 4) taken as an 

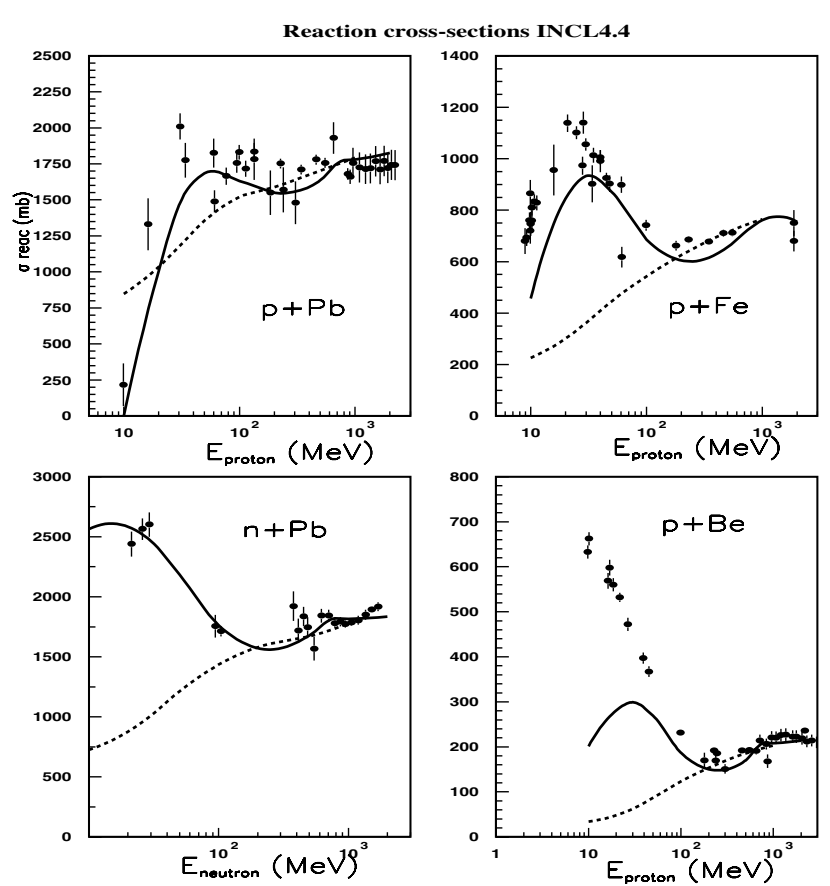

Fig. 5. Reaction cross sections as a function of the proton or neutron beam energy on various targets. Data points are a compilation [28]. The continuous line is from the new INCL4.4 calculation including coulomb distortion. The dashed line is from the previous official version INCL4.2 which had to be arbitrary re-normalized on data below $100 \mathrm{MeV}$.

example of the recent NESSI results [27]. The production of the various species are now better, especially the $\mathrm{d} /{ }^{4} \mathrm{He}$ ratio. In this approach, participant nucleons to the cascade are preferentially embedded in clusters rather than spectator ones. The consequence is a depletion of the single $20-100 \mathrm{MeV}$ proton and neutron emission however acceptable.

For sake of simplicity, the motion of particles are computed along straight lines and with a constant velocity. This can be criticized especially at the surface of the nucleus. To be more realistic, we have corrected the kinetic energy of the particle by subtraction of the nuclear potential local value. This means for example that a nucleon of Fermi energy has almost no velocity in the tail of the r-space density as it should in a classical picture. This local velocity (accordingly energy and momentum with on-shell particles) is then used for collision probabilities (reaction cross section) and kinematics. This has a large influence at the surface of the nucleus especially for a low energy of the projectile. A clear improvement is certainly the much better reaction cross section below $100 \mathrm{MeV}$ as now obtained without any re-normalization (fig. 5 continuous line). For very light nuclei, the raise of the cross section at low energy is not fully reproduced but it is probably too much outside the limits of the int ra-nuclear cascade validity. Note that at such low energies, it is crucial to correct the track of the charged projectile for Coulomb distortion.

The momentum position of the maximal $2 \mathrm{p}$-coincidence cross section in ${ }^{12} \mathrm{C}(\mathrm{p}, 2 \mathrm{p})$ at $800 \mathrm{MeV}$ is now lowered by $\sim 100 \mathrm{MeV} / \mathrm{c}$ and agrees with experimental data [29]. This is indicative of a better kinematics of collisions at the surface. Another sign is the improvement of the mean recoil velocities

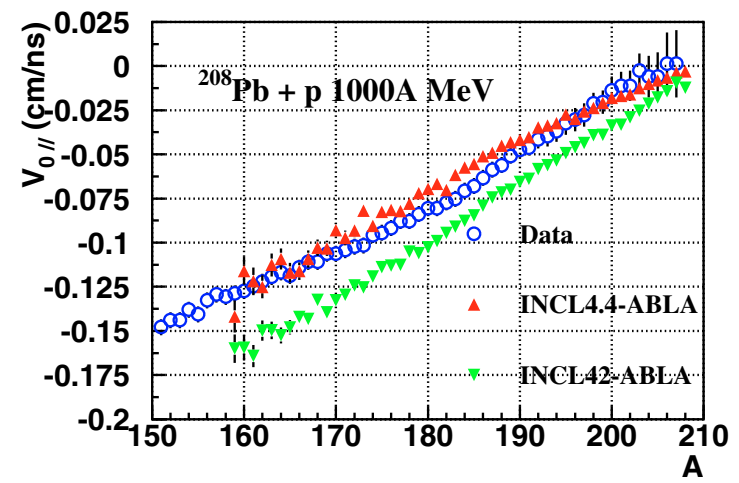

Fig. 6. Longitudinal velocity of recoil nucleus produced by a lead beam of $1 \mathrm{~A} \mathrm{GeV}$ on hydrogen expressed in the beam rest frame. Data are from [30]. Calculations show the improvement of the last INCL4.4 version compared with the official public version INCL4.2.

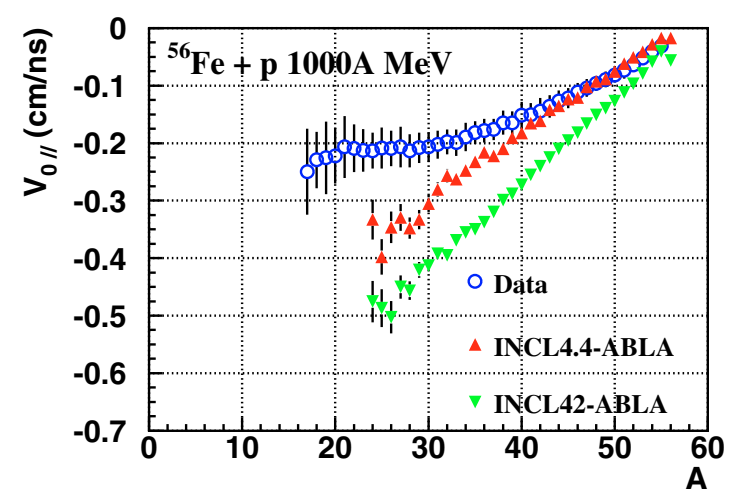

Fig. 7. Same as figure 6 but with an iron beam and data from [21].

of the evaporation residues measured at GSI in ${ }^{208} \mathrm{~Pb}+\mathrm{p}$ at $1 \mathrm{~A} \mathrm{GeV}$ and ${ }^{56} \mathrm{Fe}+\mathrm{p}$ at $1 \mathrm{~A} \mathrm{GeV}$ (figs. 6 and 7). The width of the velocity distributions (not shown) remains unchanged and very close to the experiment. The strong deviation observed for iron and recoil A values of 20-30 could be a sign of additional de-excitation mechanisms (transition states, multifragmentation) not included in the presently used version of ABLA (see [22] and [23] for details).

In figure 8 , the production of helium from iron is shown as a function of the proton beam energy. Data points are obtained in irradiation experiments ("Michel" [32]) or by direct measurements with a proton beam ("NESSI" [31] and "NESSI new" [27]) or in reverse kinematic ("SPALADIN" [22]). From the "INCL4-ABLA" to the "INCL4-Gemini" curves we can appreciate the contribution of different de-excitations (pure evaporation or addition of a transition state model). The dashed curve shows the cross section increase ( $\sim 0$ to $\sim 100 \mathrm{mb}$ ) when He production from the cascade (clusters) is included. The very recent calculation done at $1 \mathrm{GeV}$ with our last version of INCL4.4 and the last improved version of ABLA (ABLA07) shows that calculations of this important quantity is reasonable with our most recent developments but could remain $20-30 \%$ below the experimental values.

For testing the INC limits, we present the energy distributions of $\mathrm{p}, \mathrm{d}, \mathrm{t}$ and ${ }^{4} \mathrm{He}$ which are produced from neutrons of $96 \mathrm{MeV}$ on Lead (fig. 9, data from [33]) and from neutrons 


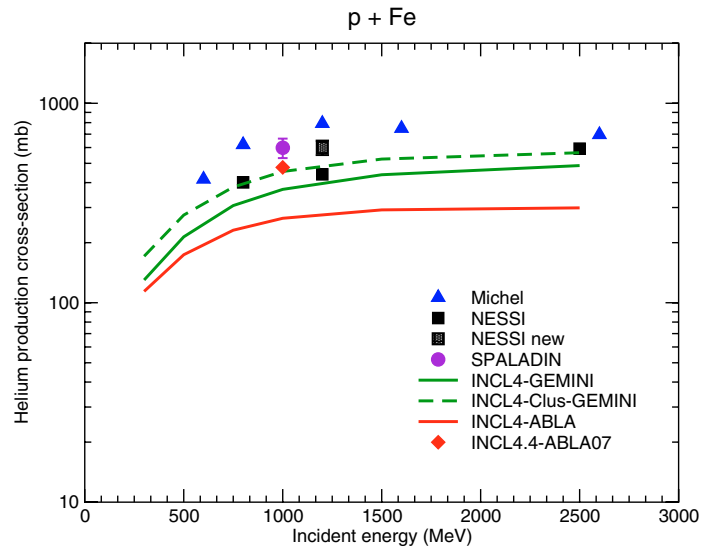

Fig. 8. Production of Helium with a proton beam of various energies on a lead target. Data points from various experiments are compared with calculations shown as lines. The diamond is also a calculation at $1 \mathrm{GeV}$ with a coupling of INCL4.4 and ABLA07.

of $63 \mathrm{MeV}$ on $\mathrm{Bi}$ (fig. 10 data from [34]). In this last case, ${ }^{3} \mathrm{He}$ and ${ }^{4} \mathrm{He}$ are summed as in the experimental detection, but ${ }^{3} \mathrm{He}$ contribution is small. At $96 \mathrm{MeV}$, results are rather good except the too high production of very low energy deuterons. This is purely due to the cascade since in ABLA only $n, p$ and ${ }^{4} \mathrm{He}$ are produced by evaporation. Actually, double differential cross sections were also measured from $20^{\circ}$ to $160^{\circ}$. The same type of agreement is observed at all angles but the calculations are decreasing with angles a bit faster than experiment. For the proton production, the quasi elastic contribution is too narrow. Whereas experimental data are smooth with energy, INCL4.4 calculations exhibits a broad peak at $20^{\circ}$ still visible at $40^{\circ}$. This was already observed in $\mathrm{p}+\mathrm{Pb}$ at $120 \mathrm{MeV}$. It is due to the local velocity correction at the surface of the nucleus which reduces the broadening of the single interaction by the Fermi motion and could be possibly corrected if diffraction would be included for outgoing particles.

The same type of defaults are observed at $63 \mathrm{MeV}$ but more pronounced (fig. 10). There is a huge production of low energy deuterons also noticeable for the tritons. The peak of protons just above the barrier is more pronounced. Very similar results are observed for $n(41 \mathrm{MeV})+\mathrm{Bi}$ for which the same type of interesting data exist [34]. These results show that our cluster procedure should be seriously improved at low energy. It is a lot too easy to aggregate a single nucleon at low energy and a procedure by elastic scattering of preformed clusters will be investigated. The precise value of Coulomb barriers and of transmission coefficients is also crucial in this domain. But the single particle production are reasonable even at these very low energies, and an acceptable link with the low energy models is possible.

Note that INCL4.2 and ABLA are already included in LAHET and MCNPX. A translation in $\mathrm{C}++$ will be available in the new release of GEANT4 and stand-alone versions with last improvements are available on request.

\section{Conclusions}

Several codes like INCL4, ISABEL, CEM2k, etc. exist and have been systematically tested for the simulation of spallation

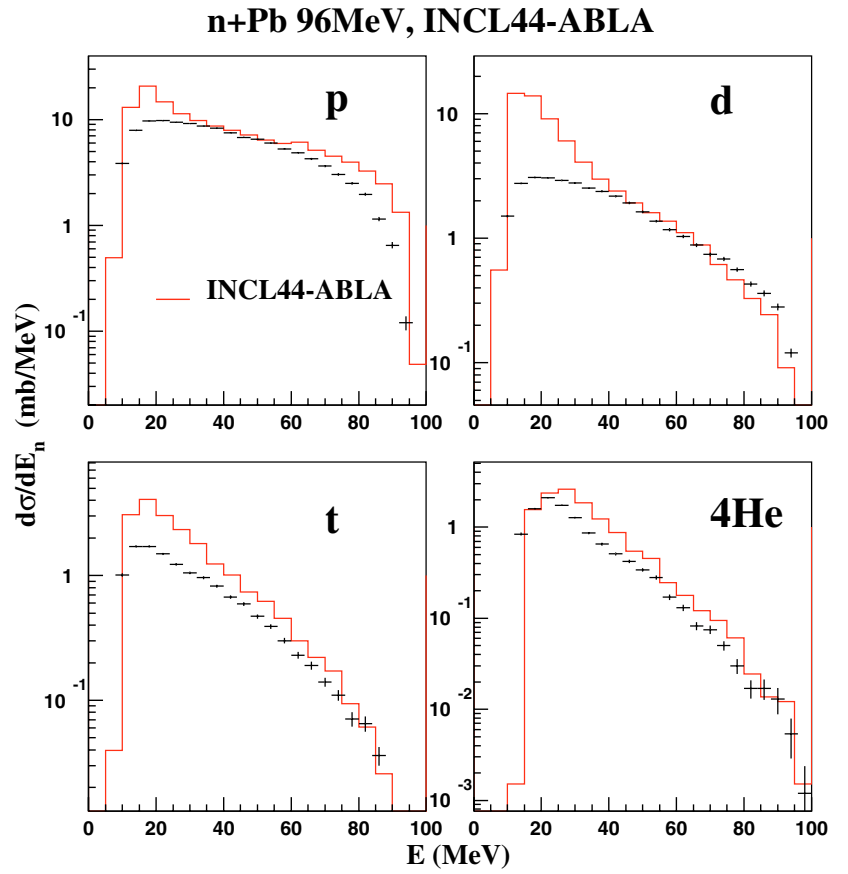

Fig. 9. Proton, deuteron, triton and $4 \mathrm{He}$ production cross sections as a function of the particle energy for a neutron beam of $96 \mathrm{MeV}$ on lead. Data points are from [33]. The histogram is an INCL4.4-ABLA calculation.

reactions in the domain $\sim 100 \mathrm{MeV}$ to a few GeV. They contain a reduced part of phenomenology which gives them a predictive power but their explicit treatment of the intra nuclear cascade can differ. It is why their comparison is useful to disentangle the most significant nuclear phenomena needed in this type of approach.

Coupled with evaporation-fission codes (like GEM or ABLA) they provide realistic generators for the production of light particles ( $\mathrm{p}, \mathrm{n}$ and pions), and of recoiling nucleus isotopically identified. The fission is also rather precisely understood especially with the ABLA code. Noticeable discrepancies however exist on the fission cross section of light elements (Ta-W region) around $1 \mathrm{GeV}$ which should be soon clarified by new experiments planned at GSI (FRS) and possibly with the ABLA07 version. Below $170 \mathrm{MeV}$, interesting recent data analyzed with CEM03 are available from TSL [35] for the fission of these target nuclei.

The systematic under-prediction of the production cross section of light fragments begins to be understood due to new experimental results like the precise analysis of the velocity spectra for intermediate masses $\left({ }^{136} \mathrm{Xe}-\mathrm{p}\right.$ experiment at FRS) and the in coincidence detection of de-excitation particles with the SPALADIN setup for the p-Fe spallation at $1 \mathrm{GeV}$. Transition states leading to asymmetric binary decays and multi-fragmentation seems to play a role but with a relative weighting not yet coherently determined. This will be probably clarified with coincidence experiments on heavier nuclei and with a computational tool like ABLA07 containing these contributions associated with the same evaporation. The experimental effort will be possible with the FAIR facility and a super-conducting magnet associated with a new TPC detector within the $\mathrm{R} 3 \mathrm{~B}$ project. 


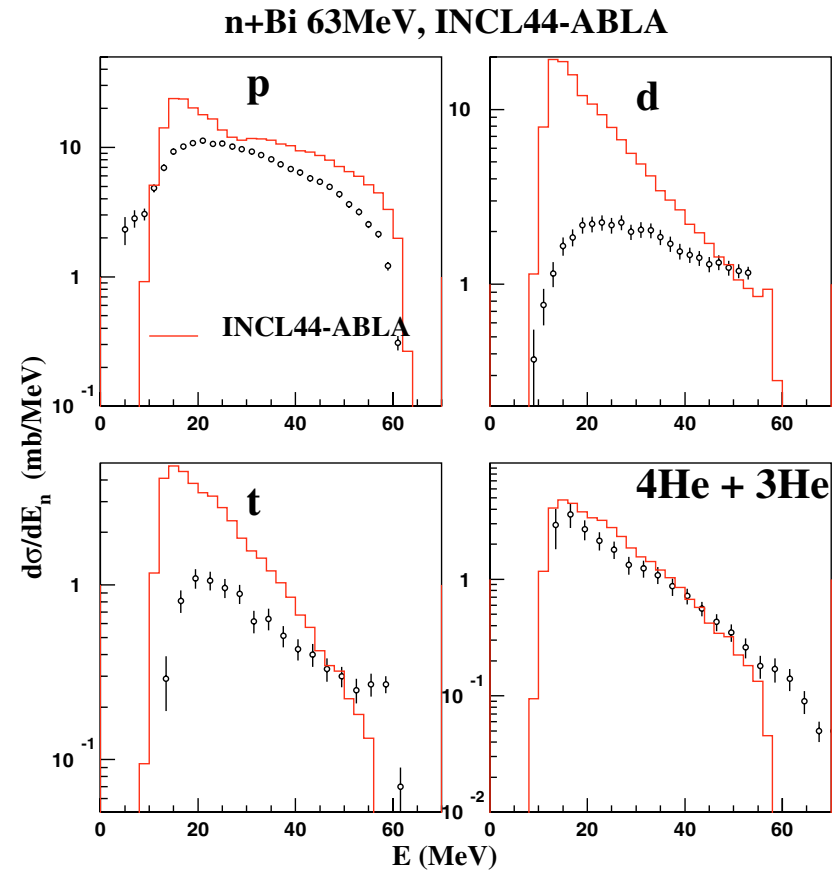

Fig. 10. Same as figure 9 but for a neutron beam of $63 \mathrm{MeV}$ on a $\mathrm{Bi}$ target. Data points are from [34].

The other critical point of the spallation models is the emission of nucleons in the range $10-50 \mathrm{MeV}$. It deals actually with the low energy validity limit of the cascade, the effective $\mathrm{NN}$ interaction and the formation of composites during the cascade. Whether or not this can be treated microscopically or by a phenomenological pre-equilibrium phase is still an open question. The refraction of low energy particles is also to be considered.

Interesting new codes or improved versions (INCL4.4, ISABEL-ETGAR, BRIC-BRIEFF, CEM03, ABLA07, etc.) appear recently and should be more systematically tested to draw clear conclusions on the physics. It will be then time to have in the community a general discussion of their very detailed and precise physical content and to compare systematically their predictions on a wide set of data now available. An intercomparison organized under the auspices of IAEA is foreseen in 2008.

To finish with, lets us remind that we have rather good and precise predictive models for spallation reactions, but they are not yet black-boxes which could be used without knowing the underlying physics and the region of possible deficiencies. For specific applications, a collaboration with developers remains frequently needed. For the future, a possible help could be the introduction of an estimated "confidence level" for each generated event produced by Monte Carlo, to be then propagated with standard techniques.
INCL (of J. Cugnon) is currently improved by Th. Aoust, A. Boudard, J. Cugnon, J.-C. David, S. Lemaire, S. Leray, P. Henrotte and Y. Yariv. ABLA is developed by K.H. Schmidt, A. Kelik, M.V. Ricciardi and J. Benlliure. We benefit also from many scientific discussions with experimentalists from Darmstadt-GSI, Jülich-COSY, OrsayIPN, University of Santiago de Compostela, etc. All of them are making this type of work possible and we thank them all.

\section{References}

1. Y. Yariv et al. (these proceedings).

2. R.J. Glauber, Lectures in Theoretical Physics (Interscience Publishers, 1959), p. 315.

3. A.K. Kerman, H. Mc Manus, R.M. Thaler, Ann. Phys. 8, 551 (1959).

4. Y. Yariv, Z. Fraenkel, Phys. Rev. C 24, 488 (1981).

5. H.W. Bertini, Phys. Rev 131, 1801 (1963).

6. J. Cugnon, Nucl. Phys. A 462, 751 (1987).

7. H. Duarte, Phys. Rev. C 75, 024611 (2007).

8. K. Niita, J. Nucl. Sci. Technol., Suppl. 2, 714 (2002).

9. Y. Watanabe, D.N. Kadrev (these proceedings).

10. M.V. Garzelli et al. (these proceedings).

11. S.G. Mashnik et al., Advance in Space Research 34, 1288 (2004).

12. S. Furihata, T. Nakamura, J. Nucl. Sci. Technol., Suppl. 2, 720 (2002).

13. J.J. Gaimard, K.H. Schmidt, Nucl. Phys. A 531, 709 (1991); A.R. Junghans et al., Nucl. Phys. A 629, 635 (1998).

14. M.V. Ricciardi et al., these proceedings; A. Kelik et al. (these proceedings).

15. R. Charity et al., Nucl. Phys A 483, 371 (1988).

16. A. Botvina et al., Nucl. Phys. 457, 633 (1987).

17. A. Boudard et al., Phys. Rev. C 66, 44615 (2002).

18. S. Leray et al., Phys. Rev. C 65, 044621 (2002).

19. S.Leray, Y. Patin et al., LNS experiment.

20. L. Audouin et al., Nucl. Phys. A 768, 1 (2006).

21. C. Villagrasa et al., Phys. Rev. C 75, 44603 (2007).

22. J.E. Ducret et al. (these proceedings); E. LeGentil, Ph.D. thesis, Évry, 2006.

23. P. Napolitani et al. (submitted to Phys. Rev.) arXiv:0706.0646.

24. Th. Aoust, J. Cugnon, Eur. Phys. J. A 21, 79 (2004).

25. J. Cugnon et al. (these proceedings).

26. A. Boudard et al., Nucl. Phys. A 740, 195 (2004).

27. C. Herbach et al., Nucl. Phys. A 765, 426 (2006).

28. R.E. Prael, M.B. Chadwick, Los Alamos Nat. Lab. preprint LA-UR-97-1744 (1997).

29. I. Tanihata et al., Phys. Lett B 100, 121 (1981).

30. T. Enqvist et al., Nucl. Phys. A 686, 482 (2001).

31. M. Enke et al., Nucl. Phys. A 657, 317 (1999).

32. R. Michel et al., Nucl. Instrum. Meth. B 103, 183 (1995).

33. V. Blideanu et al., Phys. Rev. C 70, 014607 (2004).

34. E. Raeymachers et al., Nucl. Phys. A 726, 210 (2003).

35. A.N. Smirnov et al. (these proceedings). 